University of Nebraska - Lincoln

DigitalCommons@University of Nebraska - Lincoln

Roger Kirby Publications

Research Papers in Physics and Astronomy

6-1-2004

\title{
Interactions and switching behavior of anisotropic magnetic dots
}

\author{
Kory D. Sorge \\ University of Nebraska-Lincoln, sorge@physics.fau.edu \\ Arti Kashyap \\ University of Nebraska-Lincoln, akashyap@Inmiit.ac.in \\ Ralph Skomski \\ University of Nebraska-Lincoln, rskomski2@unl.edu \\ Lanping Yue \\ University of Nebraska-Lincoln, lyue2@unl.edu \\ L. Gao \\ University of Nebraska - Lincoln \\ See next page for additional authors
}

Follow this and additional works at: https://digitalcommons.unl.edu/physics_kirby

Part of the Physics Commons

Sorge, Kory D.; Kashyap, Arti; Skomski, Ralph; Yue, Lanping; Gao, L.; Kirby, Roger D.; Liou, Sy_Hwang; and Sellmyer, David J., "Interactions and switching behavior of anisotropic magnetic dots" (2004). Roger Kirby Publications. 4.

https://digitalcommons.unl.edu/physics_kirby/4

This Article is brought to you for free and open access by the Research Papers in Physics and Astronomy at DigitalCommons@University of Nebraska - Lincoln. It has been accepted for inclusion in Roger Kirby Publications by an authorized administrator of DigitalCommons@University of Nebraska - Lincoln. 


\section{Authors}

Kory D. Sorge, Arti Kashyap, Ralph Skomski, Lanping Yue, L. Gao, Roger D. Kirby, Sy_Hwang Liou, and David J. Sellmyer 


\title{
Interactions and switching behavior of anisotropic magnetic dots
}

\author{
K. D. Sorge, ${ }^{\text {a) }}$ A. Kashyap, R. Skomski, L. Yue, L. Gao, R. D. Kirby, S. H. Liou, \\ and D. J. Sellmyer \\ Department of Physics and Astronomy and Center for Materials Research and Analysis, \\ University of Nebraska, Lincoln, Nebraska 68588
}

(Presented on 9 January 2004)

\begin{abstract}
The magnetic properties of collections of three soft magnetic nanodots with various aspect ratios are investigated. Permalloy films are first produced by dc magnetron sputtering. Focused ion beam milling is then used to mill dots, each with different shape anisotropy. We find that each of the three dots in the system has a unique switching field, and that there is significant magnetostatic coupling. Micromagnetic simulations suggest that for dot separations of less than $50 \mathrm{~nm}$ there exists strong interdot interaction, leading to the possibility of controlled switching of neighboring dots. This switching behavior is of interest in magnetic information processing. (C) 2004 American Institute of Physics. [DOI: 10.1063/1.1676053]
\end{abstract}

\section{INTRODUCTION}

In recent years, thin-film dots and dot structures have attracted much attention as scientific model systems and as precursors of advanced nanotechnologies. Thin films are rather easy to prepare by a variety of techniques such as sputtering and molecular beam epitaxy (MBE) and, once the film is created, the dots can then be formed with methods such as focused ion beam (FIB) milling or lithography. ${ }^{1-7}$

The parameter space of magnetic dot properties can be readily studied with these techniques. Using ion-beam milling allows us to study effects of dot size (both the diameter and thickness), shape effects related to the surface-to-volume ratio, shape contributions to the magnetic anisotropy, as well as different geometrical contributions to the magnetic character (such as rings ${ }^{8}$ as opposed to disks ${ }^{9}$ ). By carefully choosing the deposited film, the magnetocrystalline anisotropy (both direction and magnitude) as well as other anisotropy contributions can be tuned.

By creating multidot systems, the parameter space grows even larger and relates more closely to potential applications where many dots would be utilized. The rationale behind this work is to explore the use of magnetic nanodot arrays for logic operations. ${ }^{10-12}$ A precondition of these systems is that logic operations rely on well-defined switching states and interactions. This includes, in particular, the range of dot size and dot separation where operations can be realized without involving multidomain elements.

In this article, collections of three neighboring dots with negligible magnetocrystalline anisotropy and varying shape anisotropy are formed by sputtering permalloy $\left(\mathrm{Ni}_{80} \mathrm{Fe}_{20}\right)$ onto Si substrates and using a focused ion beam to mill the dots. Experimental investigations are coupled with micro-

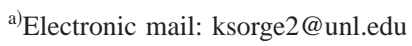

magnetic simulations for identification of parameter regions where well-defined switching and interaction effects can be expected.

\section{EXPERIMENTAL METHOD}

Thin films of permalloy were deposited on single-crystal $\mathrm{Si}$ substrates by dc magnetron sputtering. The base pressure of the system was $\approx 6 \times 10^{-7}$ Torr and argon pressure during deposition was $\approx 5$ mTorr. Films with thickness of $40-90$ $\mathrm{nm}$ were produced by sputtering at a power of $45 \mathrm{~W}$ from a premixed permalloy target.

Focused ion-beam milling with $30 \mathrm{keV} \mathrm{Ga}$ ions was used to form the dot structures. Beam currents of 11 and $70 \mathrm{pA}$ were used to create dots with sizes ranging from 150 to 500 $\mathrm{nm}$ in length and $50-150 \mathrm{~nm}$ in width. Dot separation varied from about 50 to $100 \mathrm{~nm}$, but better tailoring of the ion beam in future work should permit smaller separations. Each of the three dots was oriented with a common easy axis of shape anisotropy and arranged along the hard axis. The structures were then investigated with electron micrography and magnetic force microscopy (MFM).

\section{STRUCTURAL CHARACTERIZATION AND MICROGRAPHY}

Deposited films were checked for crystalline texture by $\mathrm{x}$-ray diffraction. Ideally, there should be no texture so that the magnetic anisotropy is only from the shape, and this was confirmed by XRD.

Once the dots were milled, the structure of the dots (or conversely, the damage to the dots from the energetic ion beam) was qualitatively analyzed. Figure 1 shows an electron micrograph of three magnetic dots with nominal thickness of $40 \mathrm{~nm}$ and length/width ratios of 400/50, 90/60, and $250 \mathrm{~nm} / 80 \mathrm{~nm}$, respectively. Atomic-force microscopy shows 


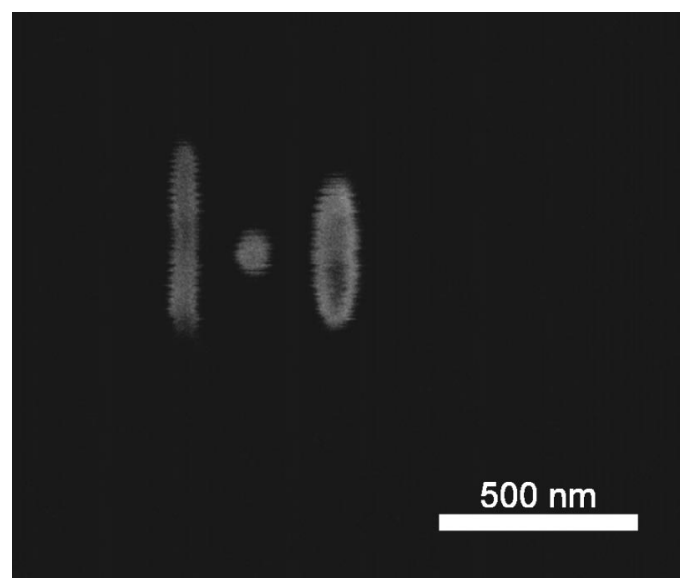

FIG. 1. Electron micrograph of permalloy dots formed on a Si substrate by sputtering, followed by ion-beam milling with a focused ion beam.

that the dots are still $\sim 40 \mathrm{~nm}$ thick at their center but stray ions from the beam mill away some of the film near the dot edges. This effect does lower the effective volume of the dots (particularly that of the highest anisotropy dot), but the dots are indeed separated.

\section{MAGNETIC PROPERTIES}

The magnetic properties of the sputtered permalloy films were measured in field with an alternating-gradient force magnetometer (AGFM) before milling. This is for additional confirmation that the material is indeed magnetically "soft" as deposited. The measured coercivity of the films was $<3 \mathrm{Oe}$.

Magnetic properties of the dots were analyzed with MFM both in field and at remanence. Figure 2 shows an MFM image of a three-dot array similar to that shown in Fig. 1 before being magnetized. From this image, it appears that the dots are very nearly single domain in their ground state.

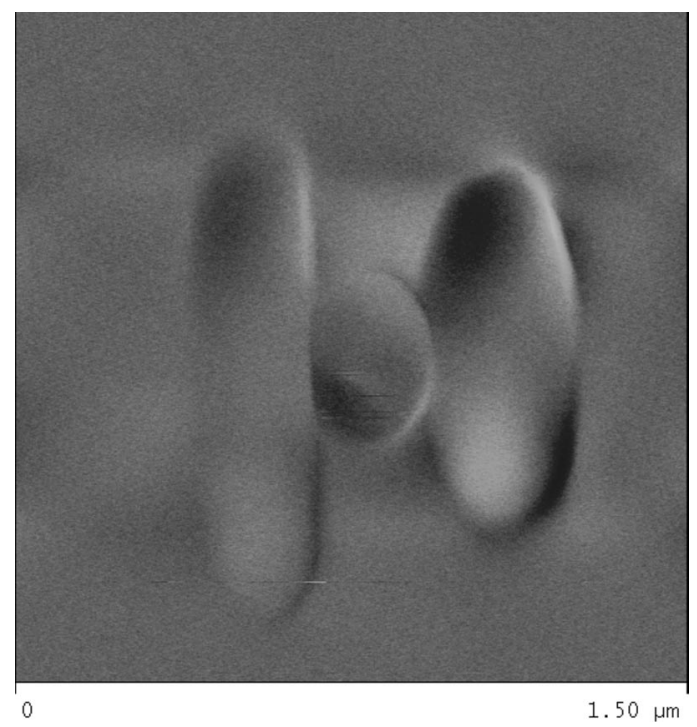

FIG. 2. MFM image of one of the three-dot arrays before being magnetized. The dots are essentially single domain but the apparent antiferromagnetic magnetostatic coupling is not always observed.

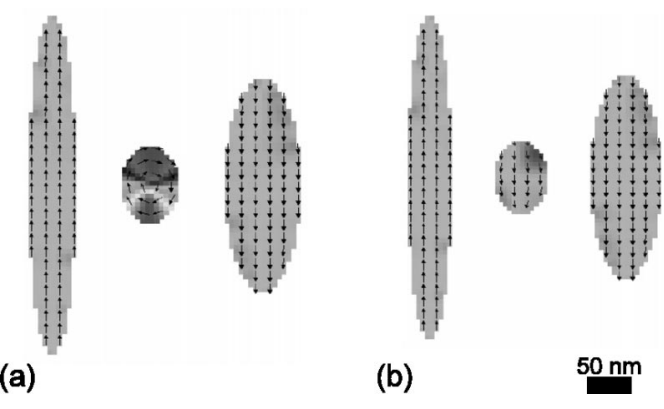

FIG. 3. Simulated three-dot spin structures: (a) multidomain center dot and (b) single-domain center dot.

This is illustrated by essentially only one "dark" spot and one "light" spot per dot in the image. In addition, there seems to be an antiferromagnetic magnetostatic coupling between the dots. This state is not always observed, but could imply that the size and separation are very near what would be required for more robust antiferromagnetic coupling of the center (magnetically soft) dot to the magnetically harder neighboring dots, if the hard dots are magnetized in the same direction.

Analysis of MFM images with field applied along the easy axes shows that the dots do have unique switching fields. Reverse fields were applied in 50 Oe steps, up to a maximum field of $400 \mathrm{Oe}$, after being saturated. One expects the switching field magnitude to be related to the shape anisotropy. We found that the small inner dot had indeed switched by 100 Oe but the only other dot that had switched by 400 Oe was the long (highest anisotropy) dot. This surprising behavior is currently being investigated.

\section{MICROMAGNETIC MODELING}

Object-oriented micromagnetic formulation (OOMMF) ${ }^{13}$ has been used to analyze the magnetic ground state of these systems. By solving the Landau-Lifshitz-Gilbert equation, the transition regimes such as the onset of equilibrium domain structures and the transition from coherent rotation to curling to more complex reversal behaviors were studied. Interactions of dots of various sizes and shapes were then analyzed more carefully. One aspect of the theoretical simulations was to identify the size, thickness, and separation ranges where FIB could be used to produce single-domain dots. This information was then used to choose the length scales discussed in Sec III. Figure 3 shows a representative system of the three dots proposed in Sec. III, with a separation of $50 \mathrm{~nm}$. When the two hard dots have the same magnetization direction (not shown in Fig. 3), the inner dot is single domain and antiferromagnetically coupled to the other dots. When the hard dots have opposing magnetization directions, the soft dot is multidomain [Fig. 3(a)]. The singledomain state can be recovered by applying a small bias field [Fig. 3(b)]. Details of these simulations will be given elsewhere.

\section{DISCUSSION AND CONCLUSIONS}

Simulation and experiment independently show that magnetic dot structures can be used to perform magnetic 


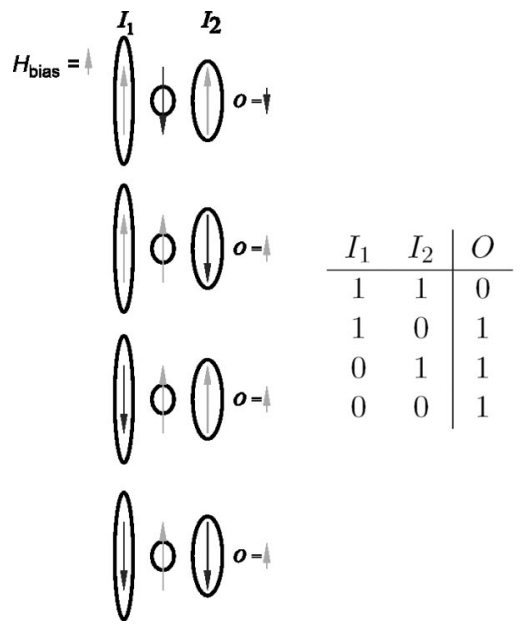

FIG. 4. Logic operation of a NAND gate based on interacting magnetic dots.

"logic" operations. By placing dots that exhibit roughly coherent magnetization reversal close enough to each other, the magnetization state of more susceptible dots can be reproducibly influenced by these neighboring dots. By having dots with different switching fields in this close proximity, external applied fields can be used to "write" the hardest dots in the system independently. Figure 4 illustrates how NAND logic could be achieved with such a system. If the two hardest dots are magnetized in the same direction, magnetostatic interactions force the soft inner dot into antiferromagnetic coupling. When the two hard dots have opposite magnetization direction, a small bias field is used to remove the interdot interaction degeneracy. The inputs of the gate are then the magnetization states of two hard dots (magnetized with external fields) and the output is the magnetization state of the soft, inner dot. Inputs and biasing can be realized through successive application of the decreasing global field magnitude: a large field to write all dots, an intermediate field to write all but the hardest dot, and a small field for biasing the low-anisotropy dot. Other logic operations could be achieved with a change in bias field, etc.

In conclusion, we have investigated systems of interacting nanodots. Motivated by the search for magnetic configurations for logic operations, we have identified the dot size, shape, and distance regimes suitable for production by FIB milling. Micromagnetic simulations indicate that welldefined switching operations and interactions can be realized for geometries and sizes accessible by focused ion-beam milling, and magnetic dot structures with the desired properties were demonstrated experimentally.

\section{ACKNOWLEDGMENTS}

This research was supported by the Army Research Office, NSF-MRSEC, the Keck Foundation, and the Center for Materials Research and Analysis.

${ }^{1}$ C.-T. Yu, D.-Q. Li, J. Pearson, and S. D. Bader, Appl. Phys. Lett. 78, 1228 (2001).

${ }^{2}$ P. Warin, R. Hyndman, J. Glerak, J. N. Chapman, J. Ferré, J. P. Jamet, V. Mathet, and C. Chappert, J. Appl. Phys. 90, 3850 (2001).

${ }^{3}$ M. Hwang, M. Shima, C. A. Ross, C. Seberino, and H. N. Bertram, J. Appl. Phys. 92, 1018 (2002).

${ }^{4}$ M. Zheng et al., Appl. Phys. Lett. 79, 2606 (2001).

${ }^{5}$ S. Y. Chou, M. Wei, P. R. Krauss, and P. B. Fisher, J. Vac. Sci. Technol. B 12, 3695 (1994).

${ }^{6}$ M. Hehn, K. Ounadjela, J. P. Bucher, F. Rousseaux, D. Decanini, B. Bartenlian, and C. Chappert, Science 272, 1782 (1996).

${ }^{7}$ R. Skomski, J. Phys.: Condens. Matter 15, R841 (2003).

${ }^{8}$ J. Rothman, M. Kläui, L. Lopez-Diaz, C. A. F. Vaz, A. Bleloch, J. A. C. Bland, Z. Cui, and R. Speaks, Phys. Rev. Lett. 86, 1098 (2001).

${ }^{9}$ R. P. Cowburn, D. K. Koltsov, A. O. Adeyeye, M. E. Welland, and D. M. Tricker, Phys. Rev. Lett. 83, 1042 (1999).

${ }^{10}$ R. P. Cowburn and M. E. Welland, Science 287, 1466 (2000).

${ }^{11}$ M. C. B. Parish and M. Forshaw, Appl. Phys. Lett. 83, 2046 (2003).

${ }^{12}$ A. Ney, C. Pampuch, R. Koch, and K. H. Ploog, Nature (London) 425, 485 (2003).

${ }^{13}$ D. G. Donahue and M. J. Porter, National Institute of Standards and Technology, Interagency Rep. No. NISTIR 6376 (1999); URL http:// www.nist.gov/oommf/ 